\section{Lungworm: what's new in management and prevention?}

\section{Robert Shiel}

Angiostrongylus vasorum infection is most common in young dogs and has been described with increased odds in certain breeds including the Jack Russell Terrier, Cocker Spaniel and Springer Spaniel. However, it can occur at any age and in any breed. The clinical presentation is variable, although signs are mostly due to effects on the respiratory, neurological or cardiovascular systems, or associated coagulopathy. Pulmonary signs are most common and include coughing, dyspnoea, tachypnoea or exercise intolerance. In such cases, crackles or increased adventitious sounds may be audible on thoracic auscultation. Haemorrhage due to coagulopathy is less common, but can be severe and rapidly fatal. For this reason, Angiostrongylus tests are often included within coagulation panels in endemic areas. Coagulopathic signs include haemoptysis, petechiae, ecchymoses, gastrointestinal bleeding, haematuria, episcleral haemorrhage or haematomas. Neurological features, such as depression, seizures, circling, ataxia, spinal pain, altered behaviour, paresis and paralysis, are usually due to central nervous system haemorrhage. Pulmonary hypertension, syncope, cor pulmonale and right-sided heart failure may occur. Minimal signs may be present prior to acute deterioration. Ocular angiostrongylosis has also been described, associated with presence of larvae or adult parasites in the anterior chamber and associated uveitis. Intraocular haemorrhage can also be present due to indirect effects. Dermal parasitic granulomas and granulomatous hepatitis have been rarely reported.

Haematology may show peripheral eosinophilia, thrombocytopenia or anaemia, or may be unremarkable. Hypercalcaemia has been reported in some animals. Coagulation profiles can show any combination of thrombocytopenia, prolonged buccal mucosal bleeding time, prolonged coagulation times, decreased fibrinogen concentration or increased fibrin degradation products or d-dimer concentrations; there is no pattern of coagulopathy that is consistently observed. Rotational thromboelastometry has shown signs consistent with hyperfibrinolysis in some cases. Bleeding may also occur in the absence of routine laboratory coagulopathy test abnormalities. Radiography often shows alveolar or interstitial patterns, especially in the peripheral lung fields or multifocally. Bronchial patterns, pleural effusion, pneumothorax and right-sided cardiomegaly may also be observed. Echocardiography may show changes consistent with pulmonary hypertension. Definitive diagnosis most commonly involves demonstration of L1 larvae in faeces (modified Baermann technique) or in bronchoalveolar lavage fluid, or the identification of antigen in blood using a patient-side test. Polymerase chain reaction-based tests are also described. Tests are routinely performed as part of the investigation of many respiratory, neurological or haemorrhagic diseases due to their relatively low cost, and the need to exclude angiostrongylosis and associated coagulopathy before pursuing more invasive tests.

$A$. vasorum is susceptible to a range of anthelmintics such as moxidectin, milbemycin oxime and fenbendazole. Supportive treatment is also necessary and may include administration of blood products, tranexamic acid, oxygen or therapy of congestive heart failure. Anti-inflammatory doses of prednisolone have been described to reduce inflammation associated with disease, or mitigate the immune response to parasite death.

\section{KEY LEARNING OBJECTIVES}

- Recognize the different clinical presentations of angiostrongylosis

- Be able to select and interpret diagnostic tests for this disease

- Be confident in the treatment of different clinical presentations

\section{MULTIPLE CHOICE QUESTIONS}

1. What is the suspected mechanism of hypercalcaemia in dogs with angiostrongylosis?
(A) Secondary hyperparathyroidism
(B) Increased activation of vitamin D
(C) Increased concentrations of parathyroid hormone-related peptide
(D) Osteolysis

2. Which of the following tests is LEAST appropriate for the confirmation of suspected brain haemorrhage associated with Angiostrongylus vasorum?
(A) Magnetic resonance imaging
(B) Computed tomography
(C) Cerebrospinal fluid analysis
(D) Antigen-based blood test

3. What radiographic finding is most likely in early Angiostrongylus vasorum infection?
(A) A peripheral alveolar pattern
(B) A perihilar alveolar pattern
(C) A generalized nodular interstitial pattern
(D) A vascular pattern 